\title{
Association between the Edinburgh CT and genetic diagnostic criteria for lobar ICH associated with cerebral amyloid angiopathy and risk of recurrent ICH
}

M Rodrigues ${ }^{1}$, L Perry ${ }^{1}$, N Samarasekera ${ }^{1}$, C Lerpiniere ${ }^{1}$, P White ${ }^{2}$, J Wardlaw ${ }^{1,3}$, R Al-Shahi Salman ${ }^{1}$

1. Centre for Clinical Brain Sciences, University of Edinburgh. 2. Institute of Neuroscience and Institute for Ageing,

Newcastle University. 3. UK Dementia Research Institute at The University of Edinburgh

\section{Background \& Aims}

- Lobar intracerebral haemorrhage $(\mathrm{ICH})$ associated with cerebral amyloid angiopathy (CAA) seems to have a higher risk of recurrent $\mathrm{ICH}$

- The Edinburgh diagnostic criteria based on CT \pm APOE genotype are accurate for diagnosing pathologicallyproven CAA-associated lobar ICH

- Whether they are associated with a higher risk of recurrent $\mathrm{ICH}$ risk in survivors of lobar $\mathrm{ICH}$ is unknown

\section{Methods}

- We conducted a 3-year, population-based, prospective inception cohort study using multiple overlapping sources of case ascertainment of ICH in 2010 to 2013 in Lothian, Scotland

- During this study, and until 2017, we recruited adults with $\mathrm{ICH}$ who consented to APOE genotyping

- A radiologist rated brain CT appearances masked to clinical, genetic and histopathological features

- Follow up (>96\% completeness) used multiple overlapping sources, and was censored at 5 years

- A neurologist validated recurrent $\mathrm{ICH}$ diagnoses and deaths using clinical and imaging records

- We estimated cumulative incidence \& performed multivariable analysis of $1^{\text {st }}$ recurrent $\mathrm{ICH}$ in participants with $11^{\text {st }}$-ever lobar $\mathrm{ICH}$ surviving at least 30 days, stratified by CT-only and CT \& APOE Edinburgh criteria (low vs intermediate/high), treating death as a competing risk

\section{Results}

- Among 120 with lobar ICH in the CT-only study, the recurrent $\mathrm{ICH}$ rate in low risk CAA was $3.0([95 \% \mathrm{Cl}$ $0.9-7.1], \mathrm{n}=42$ ) per 100 patient years versus 8.8 ([95\% Cl 5.4-13.6], $n=78$ ) for intermediate/high risk CAA

- Among 92 with lobar ICH in the CT \& APOE study, the recurrent $\mathrm{ICH}$ rate in low risk CAA was $3.2([95 \% \mathrm{Cl}$ $0.5-9.8], \mathrm{n}=23$ ) per 100 patient years versus 8.2 $([95 \% \mathrm{Cl} 4.6-13.3], \mathrm{n}=69)$ for intermediate/high risk CAA

\section{Conclusions \& future plans}

After lobar $\mathrm{ICH}$, recurrent $\mathrm{ICH}$ risk appears higher among patients with intermediate/high probability of CAA according to the Edinburgh criteria

\section{Cumulative rates of $1^{\text {st }}$ recurrent $\mathrm{ICH}$ after lobar ICH according to Edinburgh criteria}

CT only Edinburgh criteria

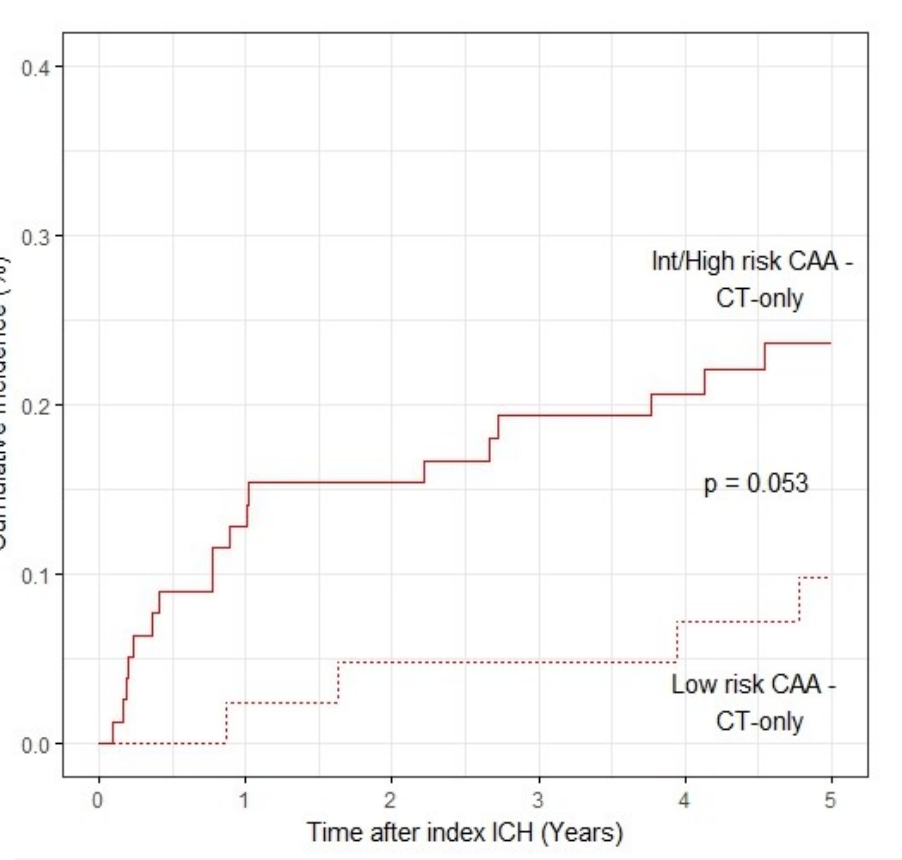

CT \& APOE Edinburgh criteria

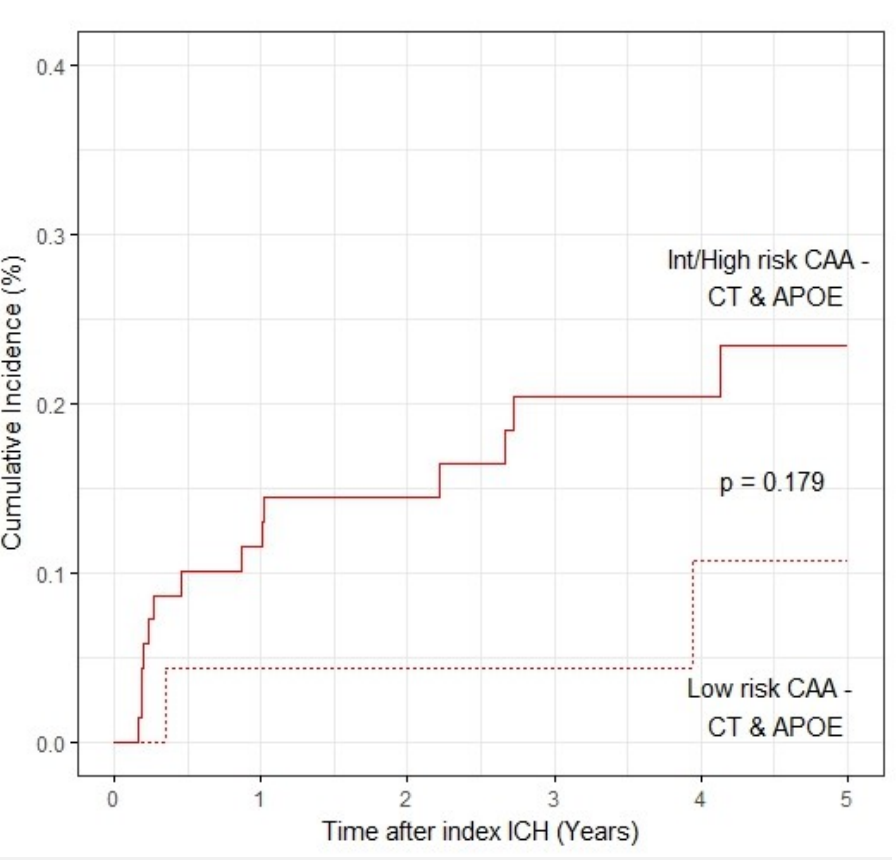

Multivariable competing risk analyses for $1^{\text {st }}$ recurrent $\mathrm{ICH}$ according to Edinburgh criteria

\begin{tabular}{|c|c|c|c|c|}
\hline & & Subhazard ratio (95 & $\% \mathrm{CI}$ & p value \\
\hline $\begin{array}{l}\text { Intermediate/hi } \\
\text { (CT-only) }\end{array}$ & gh risk CAA & $2.79 \quad(0.96-8$. & 11) & 0.06 \\
\hline Age per 10 yea & ar increase & $0.87 \quad$ (0.66-1. & 15) & 0.33 \\
\hline $\begin{array}{l}\text { Intermediate/hi } \\
\text { (CT \& APOE ) }\end{array}$ & gh risk CAA & $1.94 \quad(0.64-5$ & 94) & 0.24 \\
\hline Age per 10 yea & increase & $0.98 \quad(0.74-1$ & 29) & 0.86 \\
\hline Study flow & charts & & & \\
\hline Population-based CT-or & ly Edinburgh criteria study & Community-based CT \& & APOE Edin & rgh criteria study \\
\hline $\begin{array}{c}\text { Incident ICH } \\
n=530\end{array}$ & & $\begin{array}{c}\underset{n=359}{\text { Incident primary }} \mathrm{ICH} \\
\end{array}$ & & \\
\hline$\longmapsto$ & Secondary ICH $(n=82)$ & $\longmapsto$ & Recurre & $\mathrm{ICH}(n=14)$ \\
\hline $\begin{array}{c}\text { Primary ICH } \\
n=448\end{array}$ & & $\begin{array}{c}\text { First-ever ICH } \\
\quad n=345\end{array}$ & & \\
\hline$\longmapsto$ & Recurrent ICH (n=29) & $\longmapsto$ & No diagr & tic CT $(n=8)$ \\
\hline $\begin{array}{l}\text { First-ever ICH } \\
\quad n=419\end{array}$ & & $\begin{array}{c}\text { Diagnostic CT } \\
n=337\end{array}$ & & \\
\hline$\longmapsto$ & No diagnostic $C T(n=15)$ & $\longmapsto$ & No [ & $A(n=48)$ \\
\hline $\begin{array}{l}\text { Diagnostic CT } \\
n=404\end{array}$ & & $\begin{array}{l}\text { APOE genotype } \\
n=274\end{array}$ & & \\
\hline$\vdash$ & Non lobar ICH $(n=208)$ & $\vdash$ & Non lobe & $\mathrm{CH}(n=141)$ \\
\hline $\begin{array}{l}\text { Lobar ICH } \\
n=196\end{array}$ & & $\begin{array}{c}\text { Lobar ICH } \\
n=133\end{array}$ & & \\
\hline$\longmapsto$ & Died within 30 days $(n=76)$ & $\longmapsto$ & Died withi & 30 days $(n=41)$ \\
\hline $\begin{array}{l}\text { Included patients } \\
\quad \mathrm{n}=120\end{array}$ & & $\begin{array}{l}\text { Included patients } \\
\quad \mathrm{n}=92\end{array}$ & & \\
\hline
\end{tabular}

